\title{
Anticancer Hybrid Combinations with phenolic compounds
}

\author{
Eva María Domínguez-Martín*a,b, Ana María Díaz-Lanza*a, Patrícia Rijo ${ }^{b, c}$, Célia M. C. Faustino ${ }^{c}$ \\ aDepartment of Biomedical Sciences, Faculty of Pharmacy, University of Alcalá, Alcalá de Henares, Madrid, Spain.
}

'Research Center for Biosciences \& Health Technologies (CBIOS), Universidade Lusófona de Humanidades e Tecnologias, Campo Grande 376, 1749-024 Lisbon, Portugal.

ciMed.ULisboa - Research Institute for Medicines, Faculty of Pharmacy, Universidade de Lisboa, Av. Prof. Gama Pinto, 1649-003 Lisbon, Portugal.

E-mails: evam.dominguez@edu.uah.es; ana.diaz@uah.es

1. Introduction: Different types of treatments are been employing to overcome cancer, which is characterized by abnormal cell growth involving cell division without control. However, their usually lack of selectivity and the development of resistance result in limited efficacy or ineffectiveness of the therapies. For these reasons, the seeking of new treatment options for this disease is necessary. Nowadays, the acknowledge bioactive properties of some secondary metabolites such as polyphenols, have made antitumour hybrid combinations a promising therapeutic approach.

2. Objectives: This review provides an overview into anticancer hybrid combinations involving several phenolic compounds, focusing on their multi-target mechanisms of action and synergistic effects. It aims to contribute for the scientific validation of this type of phytopharmaceuticals as potential adjuvants in cancer (chemo)therapy.

3. Materials and Methods: Bibliographical review using the information compiled from books and electronic databases (Web of Science, PubMed and other scientific databases).

\section{Results:}

\subsection{What are anticancer hybrid combinations?}

4.4. Examples of combinations that act on different targets belonging to the same signalling pathway

Anticancer hybrid combinations are the therapeutic combination of synthetic drugs with chemically defined constituents from plants (secondary metabolites) aiming to increase the pharmacological activity of the formulation and, simultaneously, reduce the toxic side-effects of the drugs, interaction known as synergy. Example: Combinations of polyphenols with alkylating agents to enhance the activity of the drug in leukaemia cell lines.

The secondary metabolites used in these combinations are mainly plant-derived phenolic compounds and terpenoids.

Both compounds are characterized by an extensive structural diversity associated with a range of biological activities.

biological activities.
"Hybrid combination" term was introduced for the first time in 2017 by H. Wagner and T. Efferth.

Once synergistic hybrid combinations are identified, it is relevant to probe their mechanism(s) of action.

\subsection{Multi-target mechanisms}

Multi-target therapies involve the combination of different components that are not directed against a single target but instead are able to impact multiple targets simultaneously, includin enzymes, substrates, metabolites, nucleic acids, receptors, transporters, cellular organelles and/or signal cascades in a synergistic way.

Multi-target therapies are necessary for treating multifactorial diseases such as cancer.

> The main type of hybrid therapies comprises the combinations that act on different targets belonging to the same signalling pathway. This allows them to modulate multiple carcinogenic signalling to the same signalling path loops.

4.3. Scheme of the main multiple signaling pathwavs involved in apoptosis and cell cycle regulation (cellular processes often deregulated in cancer) that can be modulated by anticancer hybrid combinations:

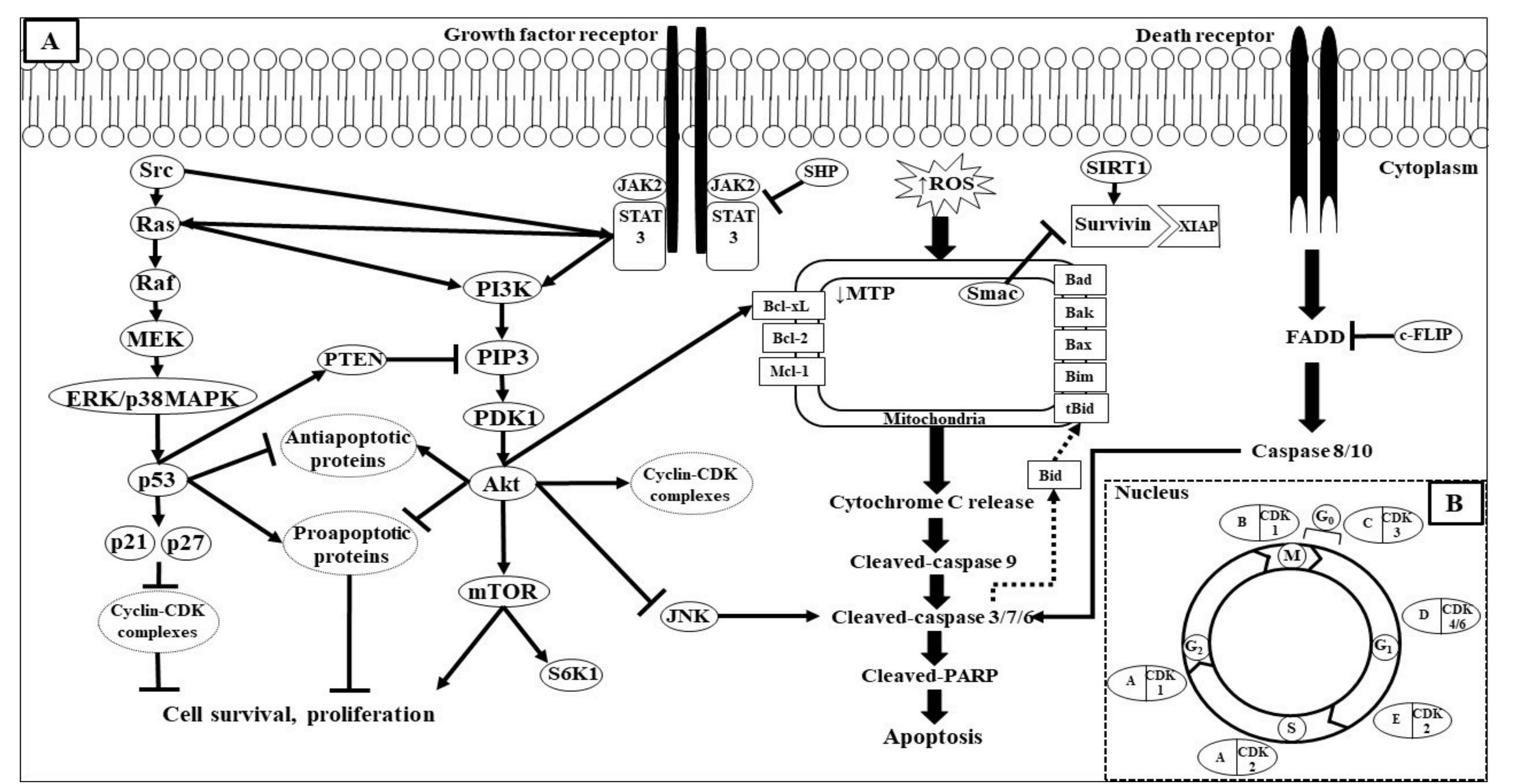

A) Schematic diagram of intrinsic and extrinsic apoptosis, and the main cellular signalling pathways affected by the hybrid combinations described. B) Representation of the cell cycle and its regulation by cyclin(A-E)cyclin-dependent kinase (CDK) complexes.

\begin{tabular}{|c|c|c|c|c|c|}
\hline $\begin{array}{c}\text { Type of } \\
\text { cancer } \\
\text { (tumoral cell } \\
\text { lines) }\end{array}$ & Phenolic compound & Drug & $\begin{array}{c}\text { Affected biological } \\
\text { process/signalling } \\
\text { pathway }\end{array}$ & $\begin{array}{l}\text { Down- } \\
\text { regulated } \\
\text { targets }\end{array}$ & $\begin{array}{c}\text { Up-regulated } \\
\text { targets }\end{array}$ \\
\hline \multirow[b]{2}{*}{$\begin{array}{l}\text { Astrocytoma } \\
\text { (MOGGCCM) }\end{array}$} & & \multirow[b]{2}{*}{ Temozolomide } & $\begin{array}{l}\text { TAutophagy } \\
(5 \mu \mathrm{M} \text { quercetin) }\end{array}$ & - & LC3-II \\
\hline & & & $\begin{array}{l}\text { Intrinsic apoptosis } \\
\text { (30 } \\
\text { MM quercetin) }\end{array}$ & $\begin{array}{l}\text { MTP, } \\
\text { HSP27/72 }\end{array}$ & $\begin{array}{l}\text { Caspase-3, Cyt } \\
\text { C }\end{array}$ \\
\hline \multirow{3}{*}{$\begin{array}{l}\text { Breast (MCF-7, } \\
\text { MDA-MB-231) }\end{array}$} & \multirow[b]{3}{*}{$\begin{array}{l}\text { Daidzein (Pueraria montana var. Lobata } \\
\text { (Willd.) Sanjappa \& Pradeep) }\end{array}$} & \multirow{3}{*}{ Centchroman } & $\uparrow$ Intrinsic apoptosis & $\begin{array}{l}\text { MTP, BCl-XL } \\
\text { in } \\
\text { MDA-MB- } \\
\text { 231 cells }\end{array}$ & $\begin{array}{l}\text { ROS, }{ }^{\text {Caspase- }} \text { Bax, } \\
\text { 9/-3/-7, PARP }\end{array}$ \\
\hline & & & Cell cycle & & $\begin{array}{l}\text { Sub-G0/G1 } \\
\text { cells }\end{array}$ \\
\hline & & & IPISK/Akt/mTOR & 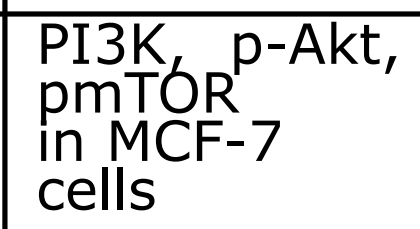 & \\
\hline $\begin{array}{l}\text { Burkitt's } \\
\text { lymphoma } \\
\text { (Namalwa, } \\
\text { Raji) }\end{array}$ & Shii & Doxorubicin & $\uparrow$ Apoptosis & |- & $\begin{array}{l}\text { Caspase-3, } \\
\text { PARP }\end{array}$ \\
\hline \multirow{3}{*}{$\begin{array}{l}\text { Gastric } \quad \text { (BCG- } \\
823)\end{array}$} & & \multirow{3}{*}{ Oxaliplatin } & IIntrinsic apoptosis & MTP & $\mathrm{ONOO}^{-}$ \\
\hline & & & $\mid$ IRaf/MEK/ERK & - & p-JNK \\
\hline & & & $\uparrow$ Autophagy & p-ULK1 & LC3-II \\
\hline \multirow{2}{*}{$\begin{array}{l}\text { Human } \\
\text { Hepatocellular } \\
\text { Carcinoma } \\
\text { (HepG2, } \\
\text { SMMC-7721) }\end{array}$} & & \multirow{2}{*}{$\begin{array}{c}\text { Doxorubicin } \\
\text { or } \\
\text { Cisplatin }\end{array}$} & TIntrinsic apoptosis & - & $\begin{array}{l}\text { PARP, } \\
\text { Caspase-3,-9, } \\
\text { Cyt C }\end{array}$ \\
\hline & & & $\uparrow$ Autophagy & - & LC3-II \\
\hline \multirow{3}{*}{$\begin{array}{l}\text { Multiple } \\
\text { myeloma } \\
\text { (LP-1, U266, } \\
\text { MM.1S, } \\
\text { MM.1R) }\end{array}$} & \multirow[b]{3}{*}{ Resveratrol (Vitis vinifera L.) } & \multirow{3}{*}{ Carfilzomib } & TIntrinsic apoptosis & $p-p 38$ & $\begin{array}{l}\text { ROS, caspase- } \\
\text { 3/- } \\
\text { 9, PARP, } \\
\text { HMOX1 } \\
\end{array}$ \\
\hline & & & Cell cycle & $\begin{array}{l}\text { p-CDK4, } \\
\text { p-cyclin D1 }\end{array}$ & G2/M cells \\
\hline & & & |ISIRT1/survivin & $\begin{array}{l}\begin{array}{l}\text { SIRT1, } \\
\text { survivin }\end{array} \\
\end{array}$ & Smac \\
\hline \multirow[b]{2}{*}{$\begin{array}{l}\text { Non-small cell } \\
\text { lung } \\
\text { cancer (A549, } \\
\text { xenograft } \\
\text { mouse model) }\end{array}$} & & \multirow[b]{2}{*}{ Docetaxel } & $\begin{array}{l}\uparrow \text { Extrinsic/intrinsic } \\
\text { apoptosis }\end{array}$ & Bid & $\begin{array}{l}\text { TRAILR-1/- } \\
3 /-4\end{array}$ \\
\hline & Chrysin (Matricaria recutita L.) & & \begin{tabular}{|l} 
Apoptosis/cell \\
proliferation
\end{tabular} & - & $\begin{array}{l}\text { p53, p21, p27, } \\
\text { IGFBP-4/-6 }\end{array}$ \\
\hline
\end{tabular}

5. Discussion and conclusion: Hybrid combinations comprising synthetic or semi-synthetic anticancer drugs and secondary metabolites from medicinal plants are a promising therapeutic strategy due to their synergistic effect and multi-target mechanisms of action. Both characteristics contribute to reduce cancer resistance to different treatments and minimize adverse effects, while simultaneously showing selectivity for tumour cells and potentiate the activity of the drug, which makes hybrid combinations an interesting prospective option to cure cancer.

Translation of anticancer hybrid combination therapy into clinical practice is therefore dependent on the development of an adequate regulatory framework and mostly on further preclinical and clinical studies.

6. References: [1] Domínguez-Martin EM, Diaz-Lanza AM, Faustino CMC (2018) Curr Pharm Des 24, 4312-4333. [2] Wagner H, Efferth T. (2017) Phytomedicine, 37, 1-3. [3] Mahbub AA, Le Maitre CL, HaywoodSmall S, Cross NA, Nicola Jordan-Mahy N (2019) Oncotarget 10, 4570-86. [4] Zhong C, Qiu S, Li J, Shen J, Zu Y, Shi J, Sui G (2019) Phytomedicine 59, 152921.

${ }^{*}$ This work is based on reference [1]. which has been updated with new published references on phenolic compounds-based anticancer hybrid combinations. 